\title{
Prompt Assessment of WST11-VTP Outcome Using Luciferase Transfected Tumors Enables Second Treatment and Increase in Overall Therapeutic Rate
}

\author{
Shimrit Fleshker ${ }^{1 \dagger}$, Dina Preise ${ }^{1 \dagger}$, Vyacheslav Kalchenko', Avigdor Scherz ${ }^{2}$ and Yoram Salomon*1 \\ ${ }^{1}$ Department of Biological Regulation, The Weizmann Institute of Science, Rehovot, Israel \\ ${ }^{2}$ Department of Plant Science, The Weizmann Institute of Science, Rehovot, Israel
}

Received 18 October 2007, accepted 24 January 2008, DOI: 10.1111/j.1751-1097.2008.00340.x

\begin{abstract}
This study hypothesized that success rate assessment of vascular targeted photodynamic therapy (VTP) of solid tumors 24 h posttreatment may allow prompt administration of a second treatment in case of failure, increasing the overall success rate. Here, we show that treatment of luciferase transfected CT26-luc mouse colon carcinoma tumors in BALB/c mice by VTP with WST11 (a Pd-bacteriochlorophyll-based photosensitizer) allows fast assessment of treatment success $24 \mathrm{~h}$ post-treatment, using bioluminescence imaging (BLI). WST11-VTP was found to abolish luciferin bioluminescence in the treated tumors resulting in two types of responses. One, comprising $75 \%$ of the mice, signified successful outcome, presenting neither BLI signal nor tumor regrowth ( $24 \mathrm{~h}-90$ days post-VTP). The second (the remaining $25 \%$ of the mice) signified treatment failure, presenting various levels of BLI signal with subsequent tumor regrowth (24 h-90 days). Consequently, the mice that failed the first treatment were treated again. We show that treatment success rate in both VTP sessions was identical and that the cumulative success rate of the treatment increased from $75 \%$ to over $90 \%$. These results therefore, present a fast method of assessing VTP outcome and support the feasibility of successive multiple treatments with these sensitizers in the clinical arena. The presented methodology can also be helpful in future preclinical studies, and expedite the development of VTP drugs.
\end{abstract}

\section{INTRODUCTION}

Vascular targeted photodynamic therapy (VTP) of solid tumors with bacteriochlorophyll (Bchl) derivatives WST09 (TOOKAD) and WST11 is based on i.v. administration/infusion of the sensitizer with concomitant local illumination of the tumor at the appropriate wavelength. Photosensitization of the circulating pigment generates cytotoxic reactive oxygen species (ROS) (1) that severely damage the illuminated tumor vasculature leading to blood occlusion (treatment end point) within minutes (2). The developing ischemia and tumor necrosis lead to tumor eradication with minimal damage to the healthy surrounding tissues (3-9).

$\dagger$ Both these authors contributed equally to this study.

*Corresponding author email: yoram.salomon@weizmann.ac.il

(Yoram Salomon)

(c) 2008 The Authors. Journal Compilation. The American Society of Photobiology 0031-8655/08
WST09 showed high success rates $(64-92 \%)$ in solid tumors, depending on tumor type and animal model. The treatment protocol consists of a single treatment session that includes 10 min of illumination $(3,5-7,10,11)$. This treatment is currently in phase II clinical trials for prostate cancer therapy $(12,13)$. WST11, a water soluble Bchl derivative, was tested in our laboratory for VTP of mouse melanoma tumors with similar cure rates (4) and is presently in phase II clinical trials for treatment of age-related macular degeneration (14). The reasons for local tumor recurrences after VTP at low but steady rates are mostly unclear. Possible explanations for this phenomenon range from vascular heterogeneity and unstable blood supply (15), temporally affecting pigment and oxygen distribution in the tumor (16-19), to inadequate delivery of light as reported for photodynamic therapy (PDT) $(20,21)$. It is likely that random pathologically related disturbances in perfusion efficiency within given tumor regions during the 5-10 min of the VTP session may temporally obstruct/deprive these sections from sufficient exposure to the cytotoxic action of ROS. Such a phenomenon may lead to the escape of the putative tumor regions from treatment, leaving potential loci for eventual local recurrence. To the best of our knowledge, there is little and mostly nonsystematic information in the literature concerning this issue.

One way to overcome the above obstacles and further improve the therapeutic rates of VTP would be to provide a second treatment session following failed treatment. The cumulative efficiency of multiple treatment sessions as a means of enhancing VTP/PDT success was only anecdotally addressed. The inherent high success rates obtained in single treatment sessions left little incentive for improvement. Furthermore, the complexity and possible ethical considerations involved with in vivo retreatment of experimental animals as well as the limited clinical applications of PDT/VTP retarded progress in this area. Only recently, preliminary evidences on repetitive in vivo aminolevulinic acid (ALA)-PDT of brain tumors for increase of treatment efficacy were reported (22).

Our approach to examine this issue took advantage of literature reports demonstrating the simplicity, reliability and speed of bioluminescence imaging (BLI) for in vivo monitoring of changes in tumor mass during development or response to antitumor therapies such as chemotherapy and PDT in preclinical studies (23-27). 
In this study, we developed an experimental tool based on BLI examination that allows determination of the treatment outcome $24 \mathrm{~h}$ after VTP in BALB/c mice bearing luciferase transfected mouse colon carcinoma (CT26-luc) tumors. In case of failure, a second treatment was given $48 \mathrm{~h}$ after the first treatment providing an increase in overall treatment success from $75 \%$ to over $90 \%$, which is a significant improvement for tumor therapy with potential clinical implications.

\section{MATERIALS AND METHODS}

Cell cultures. A cloned variant of CT26 mouse colon carcinoma cells expressing the luciferase (CT26-luc) gene was cultured in Dulbecco's modified Eagle's medium (DMEM) supplemented with $10 \%$ fetal calf serum, $2 \mathrm{~mm}$ glutamine, antibiotics $\left(100 \mu \mathrm{g} \mathrm{mL}^{-1}\right.$ penicillin and $100 \mathrm{U} \mathrm{mL}^{-1}$ streptomycin) and $1 \mathrm{~mm}$ pyruvate that was replaced every 3 days. To maintain luciferase expression, the culture medium was supplemented with $2 \mathrm{mg} \mathrm{mL}^{-1} \mathrm{G} 418$ (Cat \#345810). Cells were cultured at $37^{\circ} \mathrm{C}$ in $5 \% \mathrm{CO}_{2}$ humidified atmosphere.

Animals. Female BALB/c mice (6- to 8-week old, $\sim 20 \mathrm{~g}$ ) were housed with free access to water and food in the institutional animal facility. All experiments were conducted according to the rules of the institutional animal care and use committee.

Anesthesia. Mice were anesthetized by intraperitoneal (i.p.) injection of ketamine $10 \%$ (Rhone Merieux, Lyon, France) and xylazine 2\% (Vitamed, Hedera, Israel) mixture (85:15; vol/vol, $30 \mu \mathrm{L})$.

Analgesia. Immediately after treatment mice were i.p. injected with Flunexine $\left(2.5 \mathrm{mg} \mathrm{kg}^{-1}\right)$ and $5 \mathrm{~mL}$ of OxyCod Syrup (Rafa Laboratories Ltd, Jerusalem, Israel) was added to the water supply of the mice for 2-3 days.

Tumor model. Cultured CT26-luc monolayers were released by trypsin digestion, collected, washed once, resuspended in normal saline and injected subcutaneously (s.c.) to the leg of the mice $\left(5 \times 10^{6}\right.$ cell/mouse, $50 \mu \mathrm{L}$ ). Tumors reached treatment size of $8 \mathrm{~mm}$ diameter at about 2-3 weeks postimplantation. Tumor progression was monitored by changes in BLI and tumor volume. External caliper measurements of the length (L), width (W) and depth (D) of the tumor were taken. Tumor volume was calculated using the formula $(\mathrm{V}=\mathrm{L} / 2 \times \mathrm{W} / 2 \times \mathrm{D} / 2 \times \pi \times 4 / 3)(28)$.

Photosensitizer. The water soluble Bchl derivative WST11 (29) was provided by Steba Biotech Ltd. (Rehovot, Israel). Sensitizer concentration was determined by spectroscopy in methanol at $755 \mathrm{~nm}$, assuming $\epsilon_{0}=1.2 \times 10^{5} \mathrm{~mol}^{-1} \mathrm{~cm}^{-1}$. The pigment was dried under nitrogen and kept in the dark at $-20^{\circ} \mathrm{C}$ until used. Prior to use, the pigment was dissolved in PBS at the final concentration used for injection, as described in the specific experiments.

Light source. 4W $755 \mathrm{~nm}$ diode laser (CERAMOPTEC, Bonn, Germany) was used at a dose rate of $100 \mathrm{~mW} \mathrm{~cm}^{-2}$ for $5 \mathrm{~min}$ $\left(30 \mathrm{~J} \mathrm{~cm}^{-2}\right)$. Light was transdermally delivered as a beam using an optic fiber equipped with an FD1 frontal light distributor (Medlight S. A., Ecublens, Switzerland). The homogeneous light field on target had a diameter of $10 \mathrm{~mm} \phi$ leaving at least a $1 \mathrm{~mm}$ margin around the tumor.

Bioluminescence imaging. In vivo BLI was determined with IVIS ${ }^{\circledR}$ 100 imaging system using Living Image ${ }^{\circledR}$ software (Xenogen, CA). Mice were anesthetized and i.p. injected with $50 \mu \mathrm{L}$ D-luciferin (Cat \#XR-1001, $30 \mathrm{mg} \mathrm{mL}^{-1}$ in PBS; Xenogen). After $10 \mathrm{~min}$, one to three mice were placed on the stage inside the camera box with the tumors facing the camera. Imaging times ranged from 30 to $60 \mathrm{~s}$ in a sequential mode (two to four pictures were collected depending on tumor bioluminescence level). The IVIS ${ }^{\circledR}$ imaging system displayed light emission intensities (photons $\mathrm{s}^{-1} \mathrm{~cm}^{-2}$ radiance ${ }^{-1}$ ) as a pseudocolor overlay onto a gray scale mouse image. Maximal signal intensity was defined as the region of interest (ROI) of the tumor area quantified as photons $\mathrm{s}^{-1} \mathrm{~cm}^{-2}$ by Living Image ${ }^{\circledR}$ software. Background BLI values of equally sized nontumor ROIs of the same mouse amounted to $\leq 4-5 \times 10^{4}$ photons s $\mathrm{s}^{-1} \mathrm{~cm}^{-2}(\leq 1 \%$ of the experimental ROI) and were therefore neglected in the calculations.

PDT protocol. Anesthetized mice bearing s.c. CT26-luc tumors were i.v. injected via the tail vein with WST11 at a standard dose $\left(9 \mathrm{mg} \mathrm{kg}^{-1}\right)$ if not otherwise indicated, and immediately illuminated. Following illumination the mice were placed back in the cage to recover. The following controls were used in these experiments: dark control, i.v injection of the same sensitizer dose without illumination; light control, mice illuminated at the same light dose but no sensitizer was injected and untreated control. Tumor healing was monitored, as indicated in the specific experiments for 90 days, the time routinely defined in the laboratory for cure of tumor free mice. Mice which failed treatment were euthanized when relapsing tumors reached $\phi 12-15 \mathrm{~mm}$ as defined by the institutional animal care and use committee.

Statistical analysis. In order to describe the relationship between the bioluminescence signal and the tumor volume, we used standard regression plots. $R^{2}$ values are reported to estimate the quality of the regression model.

\section{RESULTS}

\section{Subcutaneous CT26-luc tumor growth: correlation between tumor volume and bioluminescence intensity}

The aim of this experiment was to correlate s.c. tumor volume during development as routinely determined by caliper measurements with BLI intensity. Consequently, 16 female BALB/c mice were grafted with s.c. CT26-luc tumor cells in the right or left hind leg. The tumors were visually detected within a week and further progression in growth was monitored side by side with both techniques for $\sim 2$ weeks. At least two to three measurements per tumor were taken to treatment size $(\phi \sim 8 \mathrm{~mm})$ when the mice were subjected to WST11VTP. Comparison between tumor volume $\left(\mathrm{mm}^{3}\right)$ as measured by caliper and BLI intensity throughout tumor development is presented in Fig. 1. The results show positive and significant correlation $\left(R^{2}=0.9252\right)$ between the two measurements suggesting the luciferase bioluminescence signal as a proper tool for follow-up and relative quantification of increasing tumor mass, during development with potential application for recording tumor regression.

\section{Monitoring the in vivo response of CT26-luc tumors to WST11-VTP by BLI}

The aim of this experiment was to determine the response to VTP by monitoring changes in tumor mass using BLI. Mice

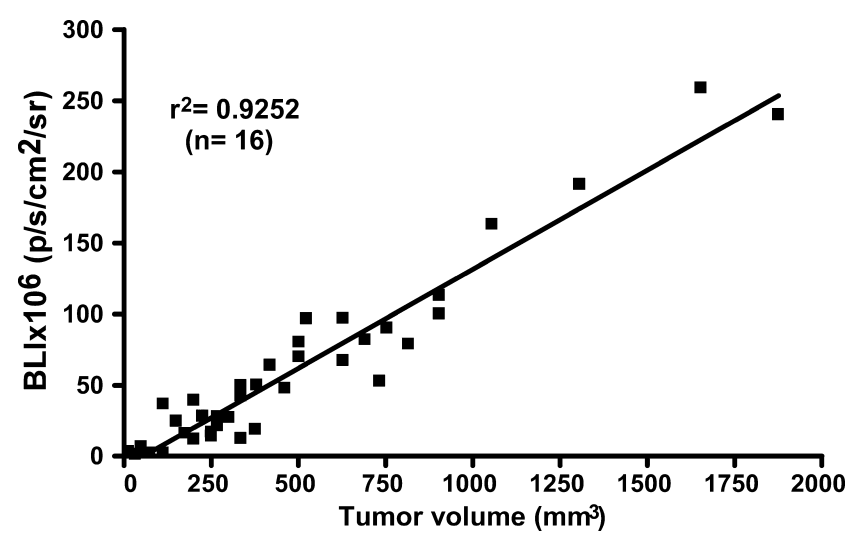

Figure 1. CT26-luc tumor growth: correlation between volume changes and bioluminescence signal intensity: CT26-luc tumors s.c. grafted to mice were subjected to two to three measurements of tumor growth by BLI and caliper methods for $\sim 2$ weeks. Calculated tumor volumes were plotted against the corresponding bioluminescence signal intensities $\left(R^{2}=0.9252, n=16\right)$. The correlation has statistical significance $(P<0.0001)$. All other details are as described in the Materials and Methods section. 
A

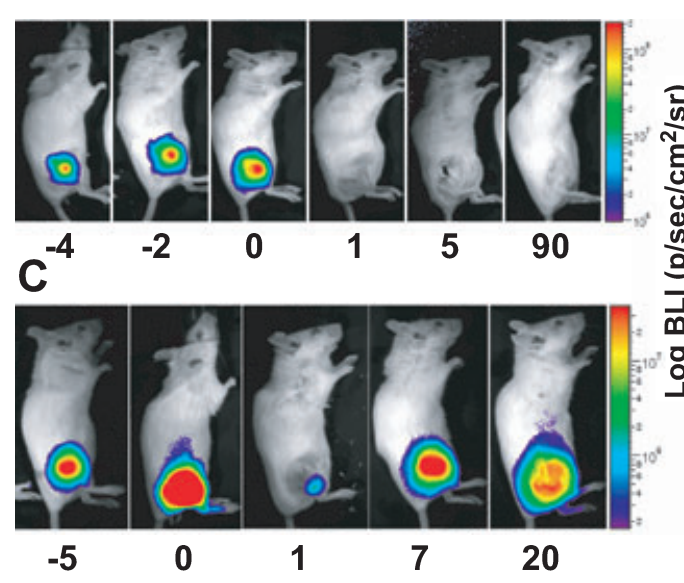

B

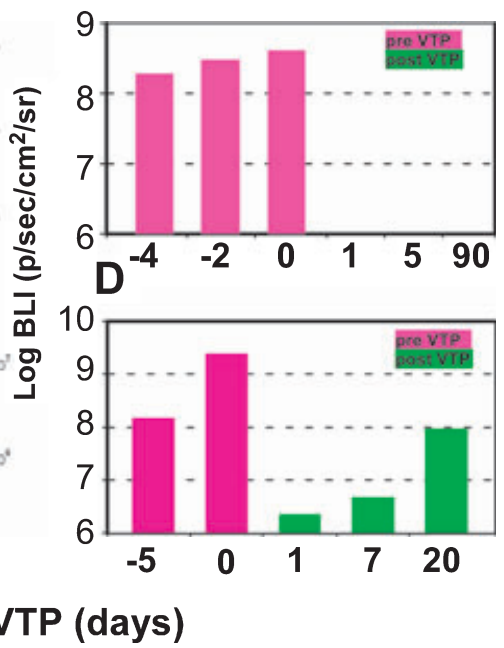

Figure 2. In vivo WST11-VTP of CT26-luc tumors: follow-up by BLI: CT26-luc tumors were subjected to standard VTP protocol and monitored by BLI at the indicated times before and after VTP. Images of representative mice are shown. (A/B) No luciferase signal was detected 24 h-90 days post-VTP (successful treatment). (C/D) Luciferase signal observed $24 \mathrm{~h}$ post-VTP with further increase at times $>24 \mathrm{~h}$. All images were set to the same pseudocolor scale to show relative bioluminescence changes over time and expressed as p s $\mathrm{cm}^{-2} \mathrm{sr}^{-1}=\left(\mathrm{photons} \mathrm{s}^{-1} \mathrm{~cm}^{-2} \mathrm{radiance}^{-1}\right)$. All other details are as described in the Materials and Methods section.

bearing CT26-luc tumors were subjected to standard VTP (WST11, $9 \mathrm{mg} \mathrm{kg}^{-1}$ ) and tumor BLI was determined on each mouse several times prior to treatment as well as at $24 \mathrm{~h}, 5-$ 7 days and up to 90 days post-VTP. A successful outcome was scored if no tumor regrowth was detected by 90 days post-VTP.

While in all mice the presence of the tumors was recorded by BLI before VTP (Fig. 2A and C, representative mice) examination at $24 \mathrm{~h}$ post-VTP clearly showed two types of responses. In one group of mice, neither BLI signal (Fig. 2A) nor local recurrences were observed during the 90 days follow-up. Such response to VTP was taken as successful (Fig. 2A and B). In contrast, the rest of the VTPtreated mice presented variable levels of bioluminescence at the treatment site $24 \mathrm{~h}$ post-VTP and an increase in BLI intensity at times $>24 \mathrm{~h}$ together with development of visible local tumor recurrences (Fig. 2C). These mice were, respectively, sacrificed when tumors developed to size limit $(\sim 12 \mathrm{~mm})$. Such response was taken as incomplete-failure (Fig. 2C and D). Visual examination of the treated tumors of both groups showed symptoms which included development of VTP-induced edema $(24 \mathrm{~h})$ and, respectively, different levels of necrosis which were visible by $\sim 48 \mathrm{~h}$ post-VTP.

In summary, a total of 53 mice were subjected to standard VTP in four independent experiments $\left(n_{1}=16, n_{2}=16\right.$, $n_{3}=7$ and $\left.n_{4}=14\right)$. Of them 40 scored no BLI signal at $24 \mathrm{~h}$, of which one died on day 30 post-VTP from an undetermined cause (with no tumor regrowth) suggesting 100\% (40/40 mice) were successfully treated. The rest (13 mice), exhibiting positive BLI signal at $24 \mathrm{~h}$ post-VTP, eventually developed local recurrences at times $<90$ days after VTP and were, respectively, euthanized (Table 1). These results suggest that BLI intensity permits accurate prediction of VTP outcome 1 day after treatment.

Consequently, the decision to deliver a second treatment in case of failure in this tumor model can be safely taken $24 \mathrm{~h}$ post-VTP. We therefore used this tool to explore the
Table 1. Bioluminescence signal in tumors following VTP: treatment outcome.

\begin{tabular}{lll}
\hline & \multicolumn{2}{c}{ Luciferase activity } \\
\cline { 2 - 3 } Time post-VTP & \multicolumn{2}{c}{+} \\
\hline $24 \mathrm{~h}$ & 40 & 13 \\
7 days & 40 & 13 \\
90 days & $39^{*}$ & $\mathrm{ND}$ \\
Therapeutic effect $n(\%)$ & $40 / 53(75)$ & $13 / 53(25)$ \\
Treatment outcome & Success & Failure \\
& & \\
\hline
\end{tabular}

*One mouse died on day 30 from an undetermined cause, without tumor regrowth. Table includes data obtained in four experiments $(n=16, n=16, n=7$ and $n=14$; total $=53) . \mathrm{ND}=$ not determined.

consequences of sequential VTP treatments and the possible benefit to the treated individual.

\section{In vivo monitoring of the response of CT26-luc tumors to second VTP upon first treatment failure}

The purpose of these experiments was to determine the therapeutic rate of a second VTP session delivered $48 \mathrm{~h}$ following treatment failure. In the first set of treatments, optimal standard VTP conditions using $9 \mathrm{mg} \mathrm{kg}^{-1}$ were sequentially applied. The first VTP session resulted in $78 \%$ success $(11 / 14)$ with only three mice $(22 \%)$ as candidates for retreatment. The three mice that failed VTP were subjected to a second treatment with the standard WST11 dose resulting in $66 \%$ success $(2 / 3)$ with a cumulative therapeutic rate of $93 \%$ (Table 2). To elevate the low degree of failure for statistical purposes a second experiment was carried out. Here, a suboptimal dose of WST11 $\left(7 \mathrm{mg} \mathrm{kg}^{-1}\right)$ was applied in the first treatment and a standard dose $\left(9 \mathrm{mg} \mathrm{kg}^{-1}\right)$ in the second (Tables 1 and 2). In two such independent experiments $\left(n_{1}=18\right.$ and $\left.n_{2}=12\right) 30$ mice were treated with $56 \%$ success $(17 / 30)$. The failed group (13/30) of mice 
Table 2. Two successive VTP treatments: individual and cumulative success.

\begin{tabular}{|c|c|c|c|c|}
\hline \multirow{2}{*}{$\begin{array}{l}\text { Time after } \\
\text { treatment }\end{array}$} & \multicolumn{2}{|c|}{$\begin{array}{l}\text { Luciferase } \\
\text { activity }\end{array}$} & \multicolumn{2}{|c|}{$\begin{array}{l}\text { Luciferase } \\
\text { activity }\end{array}$} \\
\hline & - & + & - & + \\
\hline \multirow[t]{2}{*}{ First VTP } & \multicolumn{2}{|c|}{$9 \mathrm{mg} \mathrm{kg}^{-1}$} & \multicolumn{2}{|c|}{$7 \mathrm{mg} \mathrm{kg}^{-1}$} \\
\hline & $11 / 14(78 \%)$ & $3 / 14(22 \%)$ & $17 / 30(56 \%)$ & $13 / 30(44 \%)$ \\
\hline \multirow{2}{*}{$\begin{array}{l}\text { Second } \\
\text { VTP }\end{array}$} & \multicolumn{2}{|c|}{$9 \mathrm{mg} \mathrm{kg}^{-1}$} & \multicolumn{2}{|c|}{$9 \mathrm{mg} \mathrm{kg}^{-1}$} \\
\hline & $2 / 3(66 \%)$ & $1 / 3(33 \%)$ & $10 / 13(77 \%)$ & $3 / 13(23 \%)$ \\
\hline $\begin{array}{l}\text { Cumulative } \\
\text { rate }\end{array}$ & $13 / 14(93 \%)$ & $1 / 14(7 \%)$ & $27 / 30(90 \%)$ & $3 / 30(10 \%)$ \\
\hline $\begin{array}{l}\text { Treatment } \\
\text { outcome }\end{array}$ & Success & Failure & Success & Failure \\
\hline
\end{tabular}

was subjected to the second VTP treatment under standard conditions with a success rate of $77 \%(10 / 13)$ increasing the cumulative treatment rate to $90 \%(27 / 30)$ (Table 2).

These results demonstrate an identical response rate for VTP of naïve $(40 / 53=75 \%)$ and previously treated mice $(12 / 16=75 \%)$ regardless of the treatment conditions used. Moreover, the cumulative success rates in these experiments $(90 \%$ and $93 \%)$ are significantly higher than the outcome of single treatments leaving little to improve. This result supports the view that no resistance to VTP develops within $48 \mathrm{~h}$ posttreatment.

\section{DISCUSSION}

This study was designed in order to examine the possibility of providing a second VTP treatment, following first treatment failures, and to test the principal option of increasing the overall therapeutic success with clinical implications in mind. Our results demonstrate that using the BLI method for assessment of treatment response in mice enables successful prediction of VTP outcome as early as $24 \mathrm{~h}$ post-VTP, instead of 90 days as routinely performed. To the best of our knowledge, this is the first report in which VTP outcome can be assessed that early. We showed that previously treated, as well as naïve tumors respond equally well to second treatment (Tables 1 and 2), increasing the overall therapeutic rate to $\geq 90 \%$ (Table 2); this is probably the highest success rate realistically possible.

Eradication of solid tumors treated with WST11 is achieved by vascular occlusion and blood stasis, accomplished in a single treatment session that involves a 5 min illumination. Traditionally, response to VTP, similar to other preclinical studies, is monitored by tumor volume measurements and histopathological follow-up. A new and elegant approach for monitoring tumor mass that is simple, rapid, reliable and noninvasive is provided by the BLI technology, allowing continuous follow-up of tumor development and response to treatment, by the use of luciferase transfected tumors (23-25). Yet, the method does not provide tomographic information of the examined tumor.
As part of our in vivo experiments, we established the correlation between measurements of CT26-luc tumor mass and BLI intensity (Fig. 1), confirming reports by others $(24,25)$.

In most common tumor therapies the length of the treatment protocol, the rate of tumor response and considerations of patient safety inherently extend the assessment of treatment outcome to periods of months to years. In the present study we demonstrate, for the first time, that in the case of VTP, treatment outcome can be accurately predicted within $24 \mathrm{~h}$ (Fig. 2, Table 1). This is clearly due to the very short nature of the VTP session and the rapid response of the tumor; this is accomplished by blood occlusion upon completion of the treatment protocol (2) and the exquisite sensitivity of BLI enabling detection of signals, generated even by minute numbers of luciferase transfected cells. Furthermore, BLI is absolutely dependent on the viability of the examined tissue that relies on both cellular ATP and the persistent vascular supply of luciferin and oxygen, both substrates of the luciferase reaction. Consequently, following VTP, the luciferase reaction is rapidly attenuated (minutes) while the target tumor tissue develops hypoxia, with complete elimination of the BLI signal by $7 \mathrm{~h}$ in the case of treatment success (data not shown). This leads to a void of bioluminescence signal equivalent to a nonviable tissue by $24 \mathrm{~h}$ (Fig. 2A). The high correlation of this phenomenon with the total absence of tumor relapse by 90 days post-VTP strongly supports the view that treatment success can be accurately predicted as early as $24 \mathrm{~h}$ post-treatment (Table 1). Moreover, histological examination of the treated tumor regions by 90 days confirmed the absence of residual tumor cells and/or potential local recurrences (data not shown). Likewise, it is concluded that the BLI signal following VTP predicts treatment failure and is always associated with tumor relapse (Fig. 2C and D). It is worthwhile to note that this report may be the first instance where the luciferase reaction is used for in vivo determination of acute tissue death in contrast to decline in cell numbers (24-26). Two major reasons come to mind when the mechanism underlying the disappearance of the BLI signal is considered: (i) VTPinduced vascular shutdown, inhibiting bioavailability of oxygen and luciferin and (ii) direct cellular photodamage, including luciferase inactivation and depletion of cellular ATP, as observed in the course of WST11 VTP-induced inactivation of mitochondrial membrane potential (30), or a combination thereof. Preliminary results in our laboratory suggest a major role for vascular occlusion, that may prevent luciferin and oxygen accessibility to the treated area (data not shown). The applicability of the BLI method for the general assessment of tumor response to other anticancer treatments or other tumor models using similar VTP protocols will require further research and optimization. Unfortunately, to the best of our knowledge, such sensitivity for detection of residual tumor cells is presently not available among routine imaging technologies in the clinic.

It should also be pointed out that visual examination of all treated tumors (regardless of treatment outcome) showed the same response patterns, with the development of both edema (24 h) and necrosis (48 h) post-treatment (data not shown). However, the BLI signal could uniquely differentiate the degrees of tumor response leading to the success or failure that are otherwise visually indistinguishable at these early times post-VTP. 
Chemotherapy and radiotherapy are multisession modalities in which realization of treatment outcome is a matter of months to years and retreatment, upon failure, often cannot be provided. In contrast, VTP has the advantage of being a single short-treatment modality with high success rates that apparently can be repeated, as shown here and by others for PDT (20). In preclinical studies, BLI assessment of tumor response $24 \mathrm{~h}$ post-VTP not only facilitates the process of evaluating the treatment outcome, but also expedites the decision of retreatment. The second treatment can be completed within another $24 \mathrm{~h}(48 \mathrm{~h}$ after the original treatment) with a potential increase of therapeutic rate (Table 2).

Due to the high success rate of VTP and "poor" statistics of failure (Tables 1 and 2), we intentionally selected to increase the degree of failure in the first VTP treatment session $(25 \%)$ to about $50 \%$, by applying a suboptimal photosensitizer dose ( $7 \mathrm{mg} \mathrm{kg}^{-1}$ instead of standard $9 \mathrm{mg} \mathrm{kg}^{-1}$ ) (Table 2).

Other PDT drugs (photofrin, 5-ALA) are conceivably not suitable for repetitive PDT due to higher dark toxicity, long drug-light intervals, slow clearance rates and respective cutaneous photosensitization (weeks) $(15,22,31)$. WST11 is characterized by low intrinsic toxicity and rapid clearance (hours) from the circulation (4), presenting major advantages vis à vis common PDT drugs; consequently, a second WST11-VTP treatment can be administered shortly after the first treatment, as shown here.

We also depicted that previously treated, as well as naïve tumors, respond equally well to VTP at different doses of WST11 (Table 2), suggesting that no significant resistance is induced by WST11-VTP within $48 \mathrm{~h}$. The responsiveness of tumors to a second VTP treatment and consequently elevated therapeutic rate may have several explanations: (i) following a single VTP session some tumor blood vessels are not irreversibly damaged, providing an option for a second treatment with a therapeutic rate, proportional to the sensitizer dose up to saturation. As the sensitizer dose is the limiting factor, the best solution for increasing the therapeutic rate seems to be provided by two successive treatments with optimal drug doses (32). (ii) Spontaneous local tumor relapse, following VTP, may be a consequence of temporal irregularities in blood perfusion, which is one of the major problems in solid tumor therapy. Temporal lack of perfusion may reduce oxygen and drug supply (33-35) limiting the exposure of the respective tumor regions to suboptimal cytotoxic actions of oxygen, sensitizer and light. At the same time, this must also mean that the blood supply to these initially unaffected regions is eventually renewed after completion of treatment, providing an opportunity for their subsequent BLI imaging and retreatment or development of local recurrences. In cancer therapies such as chemotherapy, the influence of random temporal variations in tumor perfusion is cancelled out by multisession treatment courses over weeks/months. In contrast, such shortterm interruptions of blood flow can be critical when the entire treatment lasts only a few minutes, as in VTP. Consequently, regions that escape treatment, due to temporal block of blood flow (36), may significantly reduce the treatment success in their vicinity, as also observed with other cancer therapies $(17,33)$. If random perfusion irregularities indeed take place the respective areas affected may become sensitive to a second VTP treatment if delivered after a short delay. Such a protocol may thus provide a potential solution for effectively treating those escaping tumor regions as well as raising the overall therapeutic effect and potential tumor control during longterm management.

In clinical cancer therapy, relapse serves as the score for treatment failure, regardless of the treatment administered. In the case of solid tumors diagnosis of treatment outcome is often based on tumor imaging or pathology and requires extended time periods of months/years. Nevertheless, it was shown that even then prostate cancer patients, who failed radiotherapy, might be subjected to VTP with TOOKAD as a second line treatment, leading to improved outcome (12). Thus, even without a rapid diagnosis of first treatment failure, a second VTP session could be successful, and possibly prolong survival. Furthermore, preclinical PDT protocols of rat sarcoma SM1 tumors consisting of four successive sessions, using a suboptimal drug dose each, resulted in overall higher success rates when compared with the cumulative drug dose administered in a single treatment session (32). Moreover, in clinical studies of skin tumors, Orenstein et al. described a PDT protocol in which two light doses were delivered within 10-15 min time interval, allowing in situ conversion of the residual prodrug (5-ALA) into the active photosensitizer, protoporphyrin, improving treatment outcome (37). Consequently, standard multiple session protocols could be suggested to patients without evaluating the outcome of each session individually. Such an approach relies on the assumption that prior VTP is not inducing resistance, in agreement with the present study whereby both treatments had similar cure rates (Table 2). Moreover, VTP with TOOKAD was able to circumvent intrinsic tumor drug resistance, due to its antivascular nature as we reported previously (5).

In the clinical trials with TOOKAD, prostate cancer response was evaluated by Gd-DTPA enhanced MRI, 7 days post-treatment. However, it should be emphasized that while being effective in assessing the extent of vascular response and estimation of the treatment volume, this method is unable to predict treatment outcome (38), which was evaluated by biopsies 6 months later (12). We have also demonstrated other imaging options for follow-up of PDT/VTP, such as diffusion weighted MRI, that can assess tumor response as early as $7 \mathrm{~h}$ post-VTP (10) and BOLD-MRI, that has the unique potential of online functional monitoring of vascular damage progression during VTP $(8,39)$. Positron emission tomography (PET) reported for evaluating PDT response $(40,41)$ and dynamic contrast enhanced perfusion CT (42) may be suggested for this purpose. However, all the above imaging techniques seem short of instantly predicting treatment outcome and are, therefore, not yet applicable for expediting the diagnostic process. The results of this study only show that the potential of prompt prediction of VTP outcome by noninvasive imaging exists and a clinical equivalent should be pursued in the future.

In summary, this study provides a new experimental approach for the prompt evaluation of tumor response to VTP, with clear advantages for an early alarm of treatment failure. Consequently, retreatment can be considered long before pathological conformation can be obtained, enabling significant elevation of the therapeutic outcome. Moreover, this technique can significantly contribute to preclinical efforts by saving time and animal lives, reducing costs of experiments, as well as expediting future development of VTP drugs. 
Unfortunately, being based on luciferase transfected tumors, this technique is not directly applicable for clinical use. Yet, tumors of laboratory animals and humans have common properties and similar response to VTP. Therefore, further studies are needed to develop new diagnostic strategies that will provide similar information about the target tumor in a clinically feasible manner and as soon as possible after treatment. An early assessment of treatment outcome may indeed enhance future effectiveness of tumor therapy for the benefit of the patients.

Acknowledgements-Y.S. and A.S. are the incumbents of the Tillie and Charles Lubin Professorial Chair in Biochemical Endocrinology, and the Robert and Yaddele Sklare Professorial Chair in Biochemistry, respectively. Submitted in partial fulfillment of MSc (S.F.) and PhD (D.P.) thesis requirements at the Fienberg Graduate School of the Weizmann Institute of Science. The authors wish to thank Ester Shai for her help. The study was supported by STEBA-BIOTECH and NEGMA (France).

\section{REFERENCES}

1. Ackroyd, R., C. Kelty, N. Brown and M. Reed (2001) The history of photodetection and photodynamic therapy. Photochem. Photobiol. 74, 656-669.

2. Madar, N. (2005) The hemodynamic basis of photosensitized BOLD MRI. MSc thesis, The Weizmann Institute of Science, Rehovot, Israel.

3. Koudinova, N. V., J. H. Pinthus, A. Brandis, O. Brenner, P. Bendel, J. Ramon, Z. Eshhar, A. Scherz and Y. Salomon (2003) Photodynamic therapy with Pd-Bacteriopheophorbide (TOOKAD): Successful in vivo treatment of human prostatic small cell carcinoma xenografts. Int. J. Cancer 104, 782-789.

4. Mazor, O., A. Brandis, V. Plaks, E. Neumark, V. RosenbachBelkin, Y. Salomon and A. Scherz (2005) WST11, a novel water-soluble bacteriochlorophyll derivative; cellular uptake, pharmacokinetics, biodistribution and vascular-targeted photodynamic activity using melanoma tumors as a model. Photochem. Photobiol. 81, 342-351.

5. Preise, D., O. Mazor, N. Koudinova, M. Liscovitch, A. Scherz and Y. Salomon (2003) Bypass of tumor drug resistance by antivascular therapy. Neoplasia 5, 475-480.

6. Schreiber, S., S. Gross, A. Brandis, A. Harmelin, V. RosenbachBelkin, A. Scherz and Y. Salomon (2002) Local photodynamic therapy (PDT) of rat C6 glioma xenografts with Pd-bacteriopheophorbide leads to decreased metastases and increase of animal cure compared with surgery. Int. J. Cancer 99, 279-285.

7. Vilensky, J., N. Koudinova, A. Harmelin, A. Scherz and Y. Salomon (2005) Vascular-targeted photodynamic therapy (VTP) of a canine-transmissible venereal tumour in a murine model with Pd-bacteriopheophorbide (WST09). Vet. Comp. Oncol. 3, 182-193.

8. Gross, S., A. Gilead, A. Scherz, M. Neeman and Y. Salomon (2003) Monitoring photodynamic therapy of solid tumors online by BOLD-contrast MRI. Nat. Med. 9, 1327-1331.

9. Zilberstein, J., S. Schreiber, M. C. Bloemers, P. Bendel, M. Neeman, E. Schechtman, F. Kohen, A. Scherz and Y. Salomon (2001) Antivascular treatment of solid melanoma tumors with bacteriochlorophyll-serine-based photodynamic therapy. Photochem. Photobiol. 73, 257-266.

10. Plaks, V., N. Koudinova, U. Nevo, J. H. Pinthus, H. Kanety, Z. Eshhar, J. Ramon, A. Scherz, M. Neeman and Y. Salomon (2004) Photodynamic therapy of established prostatic adenocarcinoma with TOOKAD: A biphasic apparent diffusion coefficient change as potential early MRI response marker. Neoplasia $\mathbf{6}$, 224-233.

11. Vakrat-Haglili, Y., L. Weiner, V. Brumfeld, A. Brandis, Y. Salomon, B. McLlroy, B. C. Wilson, A. Pawlak, M. Rozanowska, T. Sarna and A. Scherz (2005) The microenvi- ronment effect on the generation of reactive oxygen species by Pd-bacteriopheophorbide. J. Am. Chem. Soc. 127, 6487-6497.

12. Trachtenberg, J., A. Bogaards, R. A. Weersink, M. A. Haider, A. Evans, S. A. McCluskey, A. Scherz, M. R. Gertner, C. Yue, S. Appu, A. Aprikian, J. Savard, B. C. Wilson and M. Elhilali (2007) Vascular targeted photodynamic therapy with palladiumbacteriopheophorbide photosensitizer for recurrent prostate cancer following definitive radiation therapy: Assessment of safety and treatment response. J. Urol. 178, 1974-1979; discussion 1979 .

13. Weersink, R. A., A. Bogaards, M. Gertner, S. R. Davidson, K. Zhang, G. Netchev, J. Trachtenberg and B. C. Wilson (2005) Techniques for delivery and monitoring of TOOKAD (WST09)mediated photodynamic therapy of the prostate: Clinical experience and practicalities. J. Photochem. Photobiol. B 79, 211-222.

14. Valamanesh, F., M. Berdugo, R. A. Bejjani, M. Savoldelli, J. C. Jeanny, P. H. Brun, D. Blanc, D. BenEzra and F. Behar-Cohen (2004) Reduced collateral effect of photodynamic therapy (PDT) using a new water soluble photosensitizer agent. Invest. Ophthalmol. Vis. Sci. 45, E-Abstract 3188.

15. Kelleher, D. K., O. Thews, A. Scherz, Y. Salomon and P. Vaupel (2004) Perfusion, oxygenation status and growth of experimental tumors upon photodynamic therapy with Pd-bacteriopheophorbide. Int. J. Oncol. 24, 1505-1511.

16. Wang, H. W., M. E. Putt, M. J. Emanuele, D. B. Shin, E. Glatstein, A. G. Yodh and T. M. Busch (2004) Treatment-induced changes in tumor oxygenation predict photodynamic therapy outcome. Cancer Res. 64, 7553-7561.

17. Gillies, R. J., P. A. Schornack, T. W. Secomb and N. Raghunand (1999) Causes and effects of heterogeneous perfusion in tumors. Neoplasia 1, 197-207.

18. Chen, B., B. W. Pogue, I. A. Goodwin, J. A. O'Hara, C. M. Wilmot, J. E. Hutchins, P. J. Hoopes and T. Hasan (2003) Blood flow dynamics after photodynamic therapy with verteporfin in the RIF-1 tumor. Radiat. Res. 160, 452-459.

19. Minchinton, A. I. and I. F. Tannock (2006) Drug penetration in solid tumours. Nat. Rev. Cancer 6, 583-592.

20. Yu, G. Q., T. Durduran, C. Zhou, H. W. Wang, M. E. Putt, H. M. Saunders, C. M. Sehgal, E. Glatstein, A. G. Yodh and T. M. Busch (2005) Noninvasive monitoring of murine tumor blood flow during and after photodynamic therapy provides early assessment of therapeutic efficacy. Clin. Cancer Res. 11, 3543-3552.

21. Sitnik, T. M., J. A. Hampton and B. W. Henderson (1998) Reduction of tumour oxygenation during and after photodynamic therapy in vivo: Effects of fluence rate. Br. J. Cancer 77, 13861394.

22. Hirschberg, H., D. R. Sorensen, E. Angell-Petersen, Q. Peng, B. Tromberg, C. H. Sun, S. Spetalen and S. Madsen (2006) Repetitive photodynamic therapy of malignant brain tumors. $J$. Environ. Pathol. Toxicol. Oncol. 25, 261-279.

23. Rehemtulla, A., L. D. Stegman, S. J. Cardozo, S. Gupta, D. E. Hall, C. H. Contag and B. D. Ross (2000) Rapid and quantitative assessment of cancer treatment response using in vivo bioluminescence imaging. Neoplasia 2, 491-495.

24. Jenkins, D. E., S. F. Yu, Y. S. Hornig, T. Purchio and P. R. Contag (2003) In vivo monitoring of tumor relapse and metastasis using bioluminescent PC-3M-luc-C6 cells in murine models of human prostate cancer. Clin. Exp. Metastasis 20, 745-756.

25. Jenkins, D. E., Y. Oei, Y. S. Hornig, S. F. Yu, J. Dusich, T. Purchio and P. R. Contag (2003) Bioluminescent imaging (BLI) to improve and refine traditional murine models of tumor growth and metastasis. Clin. Exp. Metastasis 20, 733-744.

26. Moriyama, E. H., S. K. Bisland, L. Lilge and B. C. Wilson (2004) Bioluminescence imaging of the response of rat gliosarcoma to ALA-PpIX-mediated photodynamic therapy. Photochem. Photobiol. 80, 242-249.

27. Zeamari, S., G. Rumping, B. Floot, S. Lyons and F. A. Stewart (2004) In vivo bioluminescence imaging of locally disseminated colon carcinoma in rats. Br. J. Cancer 90, 1259-1264.

28. Sorensen, A. G., S. Patel, C. Harmath, S. Bridges, J. Synnott, A. Sievers, Y. H. Yoon, E. J. Lee, M. C. Yang, R. F. Lewis, G. J. Harris, M. Lev, P. W. Schaefer, B. R. Buchbinder, G. Barest, K. Yamada, J. Ponzo, H. Y. Kwon, J. Gemmete, J. Farkas, A. L. 
Tievsky, R. B. Ziegler, M. R. Salhus and R. Weisskoff (2001) Comparison of diameter and perimeter methods for tumor volume calculation. J. Clin. Oncol. 19, 551-557.

29. Brandis, A., O. Mazor, E. Neumark, V. Rosenbach-Belkin, Y. Salomon and A. Scherz (2005) Novel water-soluble bacteriochlorophyll derivatives for vascular-targeted photodynamic therapy: Synthesis, solubility, phototoxicity and the effect of serum proteins. Photochem. Photobiol. 81, 983-993.

30. Mazor, O. (2004) Synthesis and phototoxicity of novel sulfonate bacteriochlorophyll derivatives. PhD thesis, Weizmann Institute of Science, Rehovot, Israel.

31. Madsen, S. J., C. H. Sun, B. J. Tromberg and H. Hirschberg (2003) Repetitive 5-aminolevulinic acid-mediated photodynamic therapy on human glioma spheroids. J. Neurooncol. 62, 243-250.

32. Zhuravkin, I. N., G. Kostenich and E. A. Zhavird (1992) Photodynamic activity of chlorine e6 in experiment. Paper presented at The International Conference on Photodynamic Therapy and Medical Laser Applications, Milan, 24-27 June.

33. Abels, C. (2004) Targeting of the vascular system of solid tumours by photodynamic therapy (PDT). Photochem. Photobiol. Sci. 3, 765-771.

34. Bussink, J., J. H. Kaanders and A. J. van der Kogel (2003) Tumor hypoxia at the micro-regional level: Clinical relevance and predictive value of exogenous and endogenous hypoxic cell markers. Radiother. Oncol. 67, 3-15.

35. Chaplin, D. J., S. A. Hill, K. M. Bell and G. M. Tozer (1998) Modification of tumor blood flow: Current status and future directions. Semin. Radiat. Oncol. 8, 151-163.
36. Durand, R. E. (2001) Intermittent blood flow in solid tumours An under-appreciated source of "drug resistance." Cancer Metastasis Rev. 20, 57-61.

37. Orenstein, A., G. Kostenich and Z. Malik (1997) The kinetics of protoporphyrin fluorescence during ALA-PDT in human malignant skin tumors. Cancer Lett. 120, 229-234.

38. Haider, M. A., S. R. Davidson, A. V. Kale, R. A. Weersink, A. J. Evans, A. Toi, M. R. Gertner, A. Bogaards, B. C. Wilson, J. L. Chin, M. Elhilali and J. Trachtenberg (2007) Prostate gland: MR imaging appearance after vascular targeted photodynamic therapy with palladium-bacteriopheophorbide. Radiology 244, 196-204.

39. Tempel-Brami, C., I. Pinkas, A. Scherz and Y. Salomon (2007) Detection of light images by simple tissues as visualized by photosensitized magnetic resonance imaging. PLOS ONE 2, el191.

40. Berard, V., R. Lecomte and J. E. van Lier (2006) Positron emission tomography imaging of tumor response after photodynamic therapy. J. Environ. Pathol. Toxicol. Oncol. 25, 239-249.

41. Moore, J. V., M. L. Waller, S. Zhao, N. J. Dodd, P. D. Acton, A. P. Jeavons and D. L. Hastings (1998) Feasibility of imaging photodynamic injury to tumours by high-resolution positron emission tomography. Eur. J. Nucl. Med. 25, 12481254.

42. Meijerink, M. R., H. van Cruijsen, K. Hoekman, M. Kater, C. van Schaik, J. H. van Waesberghe, G. Giaccone and R. A. Manoliu (2007) The use of perfusion CT for the evaluation of therapy combining AZD2171 with gefitinib in cancer patients. Eur. Radiol. 17, 1700-1713. 